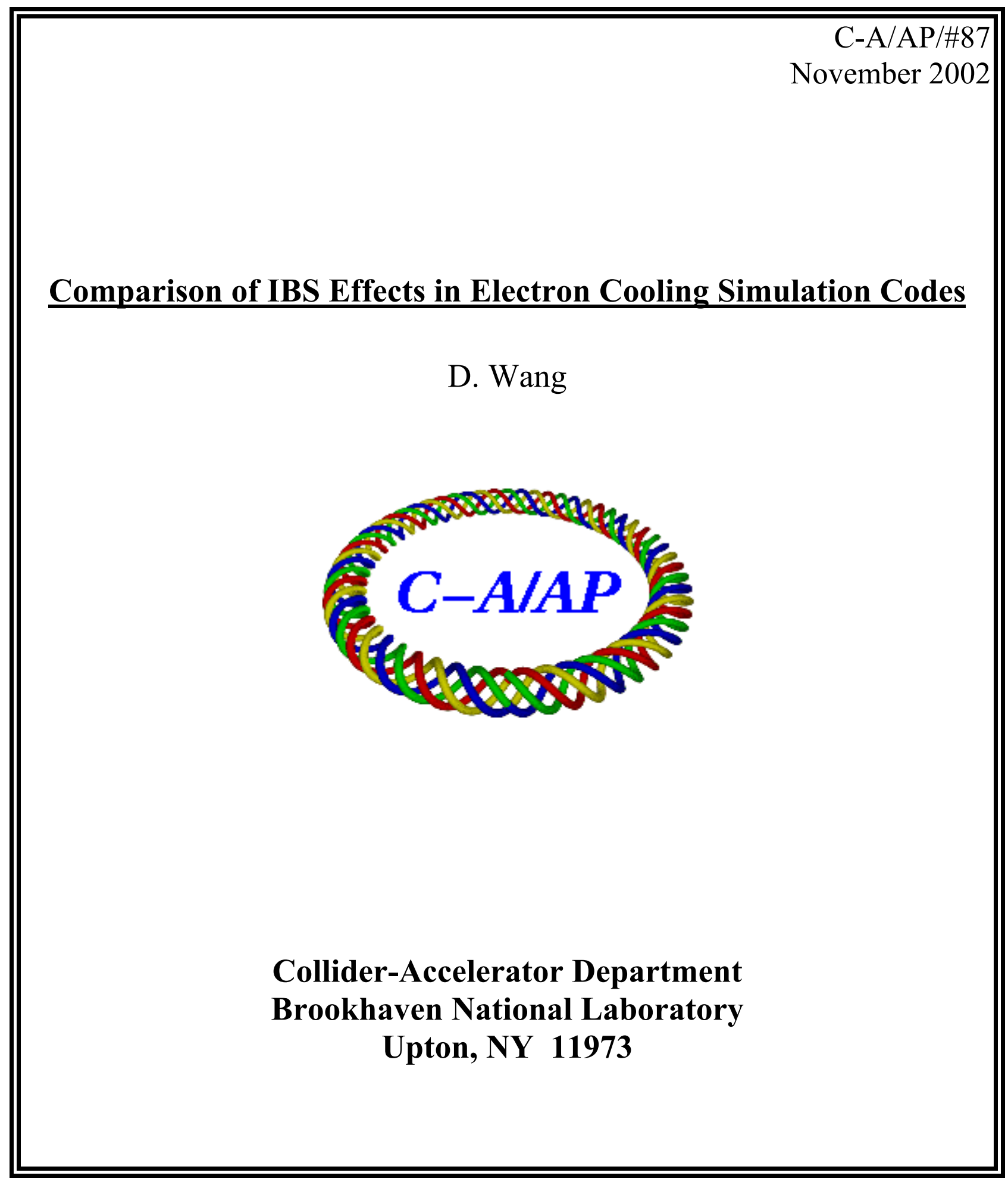




\title{
Comparison of IBS Effects in Electron Cooling Simulation Codes
}

\author{
Dong Wang \\ Collider-Accelerator Department, Brookhaven National Lab \\ Upton, NY 11973
}

Intra-Beam Scattering (IBS)[1] in the heavy particle machines leads to transverse and longitudinal emittance growth hence becomes the major effect to be overcome by the electron cooling scheme in RHIC gold beam operation to increase the luminosity.

Coulomb scattering of charged particles in a stored beam causes an exchange of energies between the transverse and longitudinal oscillations.

Intra-beam scattering is due to multiple Coulomb scattering, which leads to a diffusion in all three directions and changes, first of all, the beam dimensions.

To evaluate the effectiveness of RHIC electron cooling the IBS effect must be calculated correctly. In this paper simulations with several available IBS codes are compared,

1) Piwinski and Martini model in BETACOOL by Meshkov group in JINR

2) Parkhomchuk model in his SIMCOOL code

3) Jie Wei's model in his IBS code for RHIC, the formulae are being added to the new version of BETACOOL. 


\section{Introduction}

Intra-Beam Scattering can cause emittance growth in all dimensions and limit and performance in hadron even lepton machines. To evaluate IBS effect, different models and numerical methods are developed by Piwinski[1], Bjorken and Mtingwa[2], Martini[3], Parzen[4], Wei[5], Parkhomchuk[6] and others.

RHIC(Relativistic Heavy Ion Collider) is a double-ring collider that can operate colliding proton and ion beams at very high energy $(250 \mathrm{GeV}$ for proton and $100 \mathrm{GeV} /$ nucleon for ion).

Table 1, Nominal RHIC ion beam parameters used in the IBS simulations

\begin{tabular}{|c|c|}
\hline Charge & 79 \\
\hline Atomic number & 197 \\
\hline Energy & $100 \mathrm{GeV} /$ nucleon \\
\hline Gamma & 107.5 \\
\hline Bunched/coasting & Bunched \\
\hline Number of ion/bunch & $1 \times 10^{9}$ \\
\hline Initial beam emittance $(95 \%)$ & $15 \pi \mathrm{mm} . \mathrm{mrad}$ \\
\hline Initial bunch length (rms) & $30 \mathrm{~cm}$ \\
\hline Initial energy spread & $1 \times 10^{-3}$ \\
\hline Transverse coupling & Full coupling \\
\hline
\end{tabular}

The simulations described in this report will be carried out with above RHIC parameters. In some codes in which different conventions are adopted the conversions are made to ensure that the same physical parameters are meant.

Below the IBS models in three major computer simulation codes are briefly mentioned. The detailed comments on those analytical or semi-analytical models are beyond scope of this paper.

\section{1, IBS models in BETACOOL(Martini and Piwinski model)}

BETACOOL[7] is comprehensive simulation code developed in JINR(Joint Institute of Nuclear Research, Russia) that is able to calculate many physical phenomena in the storage rings, including 
a) Intra-Beam Scattering

b) Electron cooling

c) Stochastic cooling

d) Scattering on residual gas

e) Other heating process

f) Injection

g) Interaction with internal target, etc.

Two well-known models, the Piwinski model[1] and Martini model[3] are adopted in IBS calculations in BETACOOL. The latter option in the BETACOOL requires the lattice functions along the ring. In our calculation the RHIC FODO structures are included which is a good approximation.

\subsection{Rate with different simulation steps in BETACOOL}

First we try to determine the right number of iteration steps for accurate calculations in a reasonable time period. Generally speaking, Piwinski model in BETACOOL is simpler hence it takes much less time. In the comparison below, we choose the number of steps as 1000 .

Table 2, Determine the steps of Piwinski model option

\begin{tabular}{|c|c|}
\hline Piwinski model (x/y/s) & Steps \\
\hline $2.2 \mathrm{E}-6 /-3.4 \mathrm{E}-8 / 9.9 \mathrm{E}-7$ & 100 \\
\hline $2.2 \mathrm{E}-6 /-3.8 \mathrm{E}-8 / 9.6 \mathrm{E}-7$ & 1000 \\
\hline $2.2 \mathrm{E}-6 /-3.8 \mathrm{E}-8 / 9.6 \mathrm{E}-7$ & 10000 \\
\hline
\end{tabular}

Martini model takes into account the RHIC beam optics. The fact that RHIC is a large machine with thousands of elements results in the much longer computing time for its IBS calculations. In the comparison below, we choose the number of steps as 100 .

Table 3, Determine the steps of Martini model option

\begin{tabular}{|c|c|}
\hline Martini model (x/y/s)(coupled) & Steps \\
\hline $5.0 \mathrm{E}-5 / 5 \mathrm{E}-5 / 6.8 \mathrm{E}-5$ & 10 \\
\hline $7.9 \mathrm{E}-5 / 7.9 \mathrm{E}-5 / 9.7 \mathrm{E}-5$ & 100 \\
\hline $8.1 \mathrm{E}-5 / 8.1 \mathrm{E}-5 / 9.9 \mathrm{E}-5$ & 500 \\
\hline
\end{tabular}

1.2 Comparison of with Piwinski and Mardini model in BETACOOL

The comparison is made and the significant discrepancy is found between the results from two major models in the BETACOOL code. 
Table 4, Initial IBS Rate under typical RHIC parameters

\begin{tabular}{|c|c|c|c|c|}
\hline Bunch L & Piwinski model (x/y/s) & Steps & Martini model (x/y/s) & Steps \\
\hline $20 \mathrm{~cm}$ & $3.3 \mathrm{E}-6 /-5.8 \mathrm{E}-8 / 1.5 \mathrm{E}-6$ & 1000 & $2.4 \mathrm{E}-4 /-3.7 \mathrm{E}-6 / 1.5 \mathrm{E}-5$ & 100 \\
\hline $30 \mathrm{~cm}$ & $2.2 \mathrm{E}-6 /-3.8 \mathrm{E}-8 / 9.6 \mathrm{E}-7$ & 1000 & $7.9 \mathrm{E}-5 /-7.9 \mathrm{E}-5 / 9.7 \mathrm{E}-5$ & 100 \\
\hline $60 \mathrm{~cm}$ & $1.1 \mathrm{E}-6 /-1.9 \mathrm{E}-8 / 4.9 \mathrm{E}-7$ & 1000 & $8.1 \mathrm{E}-5 /-1.3 \mathrm{E}-6 / 4.9 \mathrm{E}-5$ & 100 \\
\hline $100 \mathrm{~cm}$ & $6.4 \mathrm{E}-7 /-1.1 \mathrm{E}-8 / 2.9 \mathrm{E}-7$ & 1000 & $4.8 \mathrm{E}-5 /-7.3 \mathrm{E}-7 / 2.9 \mathrm{E}-5$ & 100 \\
\hline
\end{tabular}

From Table 4 one can see that the discrepancy is so large that one of two models must be wrong. The experiments show that the growth time for RHIC ion beams due to the Intra-Beam Scattering effect is within the order of hours. As the result the Piwinski model that is far away in this case is not chosen for the comparison below.

\section{2, Jie Wei's formulae and code}

In reference[5], formulae are derived under certain assumptions to improve the accuracy and the speed of the calculation. The formulae take into account the ring optics and treat the issue in high energy and low energy in different ways. Those formulae are developed in BNL and aiming at the use on the RHIC. The bunch-marking and comparison with experiments are performed. So this model is considered promising for the IBS and cooling evaluations for RHIC. Now this code is a stand-alone program and is being included into the BETACOOL code. The growth times from intra-beam scattering are computed for the case of FODO cells only, which is a good approximation for the RHIC.

The rise times in transverse dimension are given as

$$
\begin{aligned}
& {\left[\begin{array}{c}
\tau_{x} \\
\tau_{y}
\end{array}\right]=\left[\begin{array}{c}
\frac{1}{\sigma_{x}} \frac{d \sigma_{x}}{d t} \\
\frac{1}{\sigma_{y}} \frac{d \sigma_{y}}{d t}
\end{array}\right]} \\
& =\frac{Z^{4} N_{b}}{A^{2}} \frac{r_{0}^{2} L_{c}(\beta c)}{8 \pi(\beta \gamma) \varepsilon_{x} \varepsilon_{y} \varepsilon_{z}} F(\chi)\left[\begin{array}{c}
-a^{2} / 2+d^{2} \\
-b^{2} / 2
\end{array}\right]
\end{aligned}
$$


The longitudinal rise time is

$$
\begin{aligned}
& \tau_{\text {long }}^{-1}=\frac{1}{\sigma_{\text {long }}} \frac{d \sigma_{\text {long }}}{d t} \\
& =\frac{Z^{4} N_{b}}{A^{2}} \frac{r_{0}^{2} L_{c}(\beta c)}{8 \pi(\beta \gamma) \varepsilon_{x} \varepsilon_{y} \varepsilon_{z}} F(\chi)\left(1-d^{2}\right)
\end{aligned}
$$

where $\sigma_{\text {long }}$ denotes the longitudinal rms bunch length, $t$ time, $Z$ and $\mathrm{A}$ charge and mass number of the ions respectively, $\mathrm{N}_{\mathrm{b}}$ the number od particles in the bunch, $\mathrm{r}_{0}$ is the classical electron radius, $\mathrm{L}_{\mathrm{c}}$ is the logarithm, $\beta$ and $\gamma$ the relativistic factors, c the speed of light. $\varepsilon_{x}, \varepsilon_{y}, \varepsilon_{z}$ are the normalized transverse and longitudinal rms emittances.

In case the vertical dispersion can be neglected, one has

$$
\begin{aligned}
& d=\frac{D_{x} \sigma_{p}}{\left(\sigma_{x}^{2}+D_{x}^{2} \sigma_{p}^{2}\right)^{1 / 2}} \\
& a=\frac{\beta_{x} d}{D_{x} \gamma}, b=\frac{\beta_{y} \sigma_{x}}{\beta_{x} \sigma_{y}} a, \chi=\frac{a^{2}+b^{2}}{2}
\end{aligned}
$$

where $D_{x}$ is the horizontal dispersion, $\sigma_{x, y, p}$ the transverse and momentum rms beam sizes repectively, and $\beta_{x, y}$ the transverse $\beta$-functions.

The $\chi$ is defined by

$$
F(\chi)=\frac{(1+2 \chi) I(\chi)-3}{1-\chi}
$$

with

$$
I(\chi)=\left\{\begin{array}{l}
\frac{1}{\sqrt{\chi(\chi-1)}} \arctan h \sqrt{\frac{\chi-1}{\chi}}, \chi \geq 1 \\
\frac{1}{\sqrt{\chi(1-\chi)}} \arctan \sqrt{\frac{1-\chi}{\chi}}, \chi<1
\end{array}\right\}
$$

Figure 1 and Figure 2 give the typical results of IBS calculations for RHIC with Jie Wei's code. Figure 1 shows the transverse emittance evolution with different particle numbers per bunch. Figure 2 is about the bunch length vs. time. 
IBS Calculation by IBS-JW

Nominal RHIC Paramters

Emittance vs. Number of lons per bunch

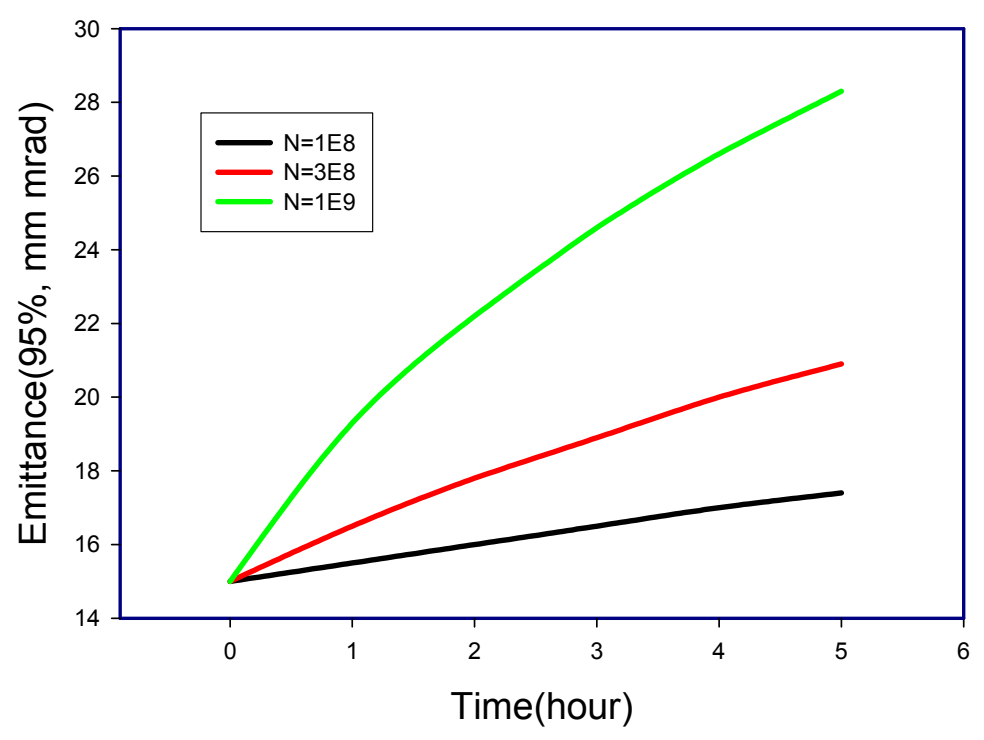

Figure 1

IBS Calculation by IBS-JW

Nominal RHIC Parameters

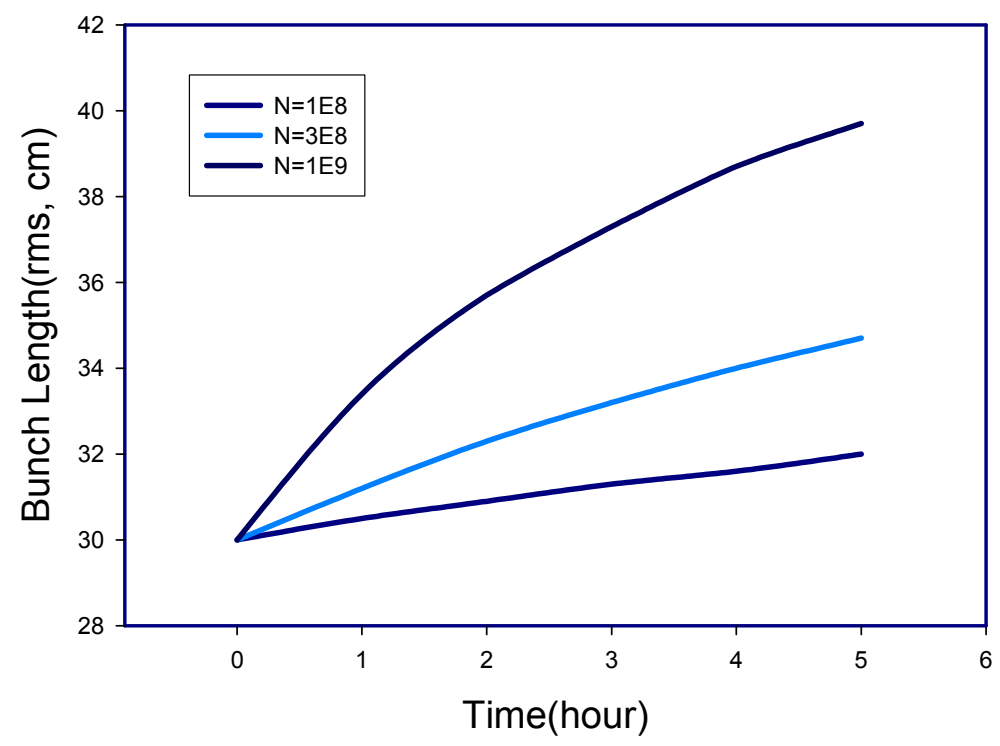

Figure 2 


\section{3, The benchmarking of Jie-Wei's code with experiments on RHIC}

In reference[8] a comparison between simulation with Jie Wei's code and some preliminary experiments in RHIC machine is made. The observations are

1) Transverse emittance, experiment shows a faster growth than that in simulation. However there are still some uncertainties in the measurements. Several factors may affect the experimental results.

2) Longitudinal dimension, good agreement is seen between the simulation and the experiment.

Up to now, most experiments are performed in relatively short time periods and with colliding beams. Further experiments are being planned to get a better understanding on the IBS issue in the RHIC.

\section{4, Parkhomchuk's model in SIMCOOL}

SIMCOOL is a fast code written by V. Parkhomchuk to calculate the IBS and electron cooling effect. The IBS simulation in SIMCOOL is based on a semi-phenomenological model introduced by Parkhomchuk[6].

The definition of rise time in the SIMCOOL

$$
\frac{1}{\tau_{I B S}}=\frac{1}{\left\langle\Delta p^{2}\right\rangle} \frac{d\left\langle\Delta p^{2}\right\rangle}{d t}
$$

The semi-phenomenological model is made as

$$
\frac{1}{\tau_{I B S}}=\frac{4 \pi\left(Z_{i} e\right)^{4} n_{i} L n_{I B S}}{M_{i}^{2}\left\langle V^{3}\right\rangle}
$$

and

$$
n_{i}=\frac{N_{i}}{2 \pi \varepsilon_{\perp} \beta_{\perp} l_{b} \gamma}
$$

where $\sigma_{\text {long }}$ denotes the longitudinal $\mathrm{rms}$ bunch length, $t$ time, $Z$ charge number of the ions, $\mathrm{N}_{\mathrm{i}}$ the number of particles in the bunch, $\mathrm{r}_{0}$ is the classical electron radius, $\mathrm{L}_{\text {nibs }}$ is the logarithm(chosen as 10 in the code), $\beta$ and $\gamma$ the relativistic factors, $\mathrm{c}$ the speed of light. $\varepsilon_{\perp}$, is the normalized transverse rms emittance. 
The model was assumed to work with a simple uniform(square) particle distribution in a bunch. In SIMCOOL the particle distribution in the ion or proton bunch may vary significantly during the simulation due to the IBS or electron cooling effects. The tails can develop therefore the applicability of the IBS model becomes questionable. In order to avoid this problem the FWHM(Full Width Half Maximum) is adopted as the measure of the change of bunch dimensions in our SIMCOOL simulation.

\section{4, Comparison of simulations with 3 major IBS models}

Calculations for the comparison are done with three models, say, Martini model option in the BETACOOL, Parkhomchuk's model in the SIMCOOL, the Jie Wei's model. The same set of RHIC parameters are used. The time period is chosen as 5 hours. The figure $3,4,5$

Comparison of RHIC IBS Calculations

by JW-IBS, BETACOOL and

SIMCOOL(scaled from FWHM)

Tran. Emittance vs. Time

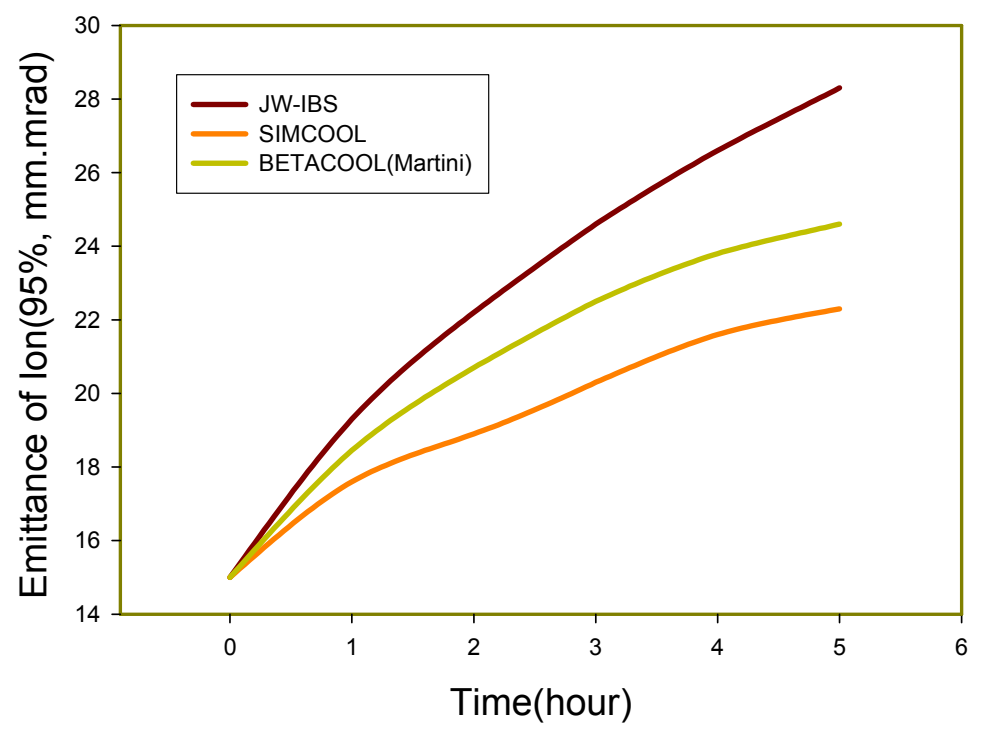

Figure 3 
Comparison of RHIC IBS calculations

by JW-IBS and BETACOOL(Martini model)

Bunch length vs. Time

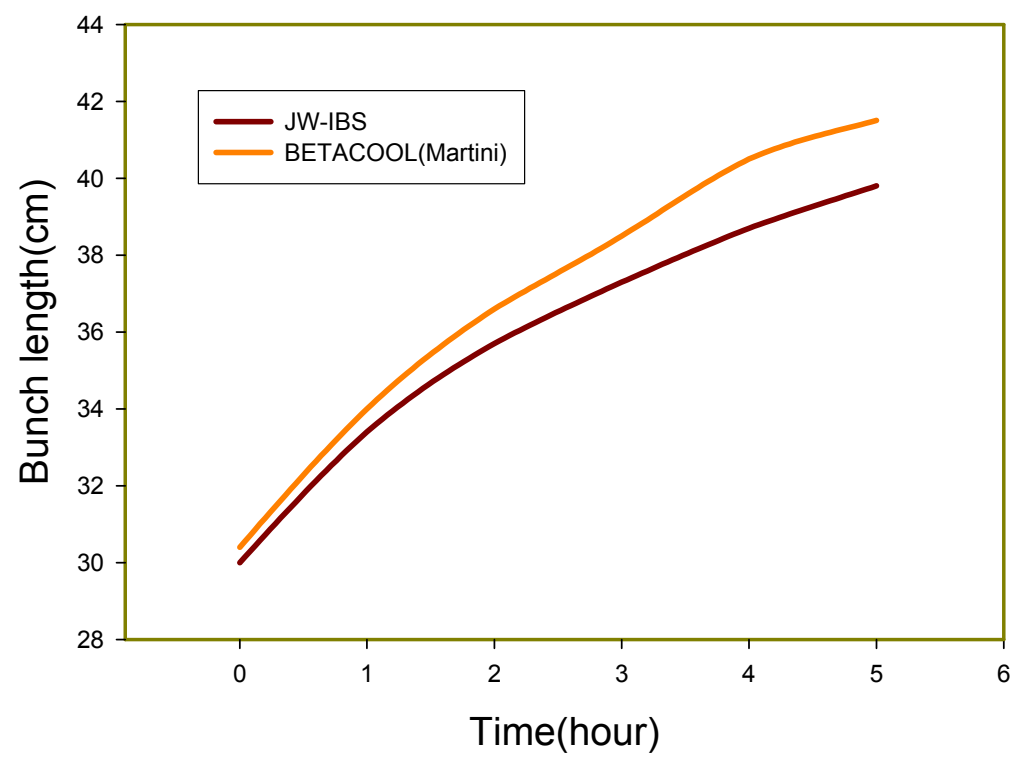

Figure 4
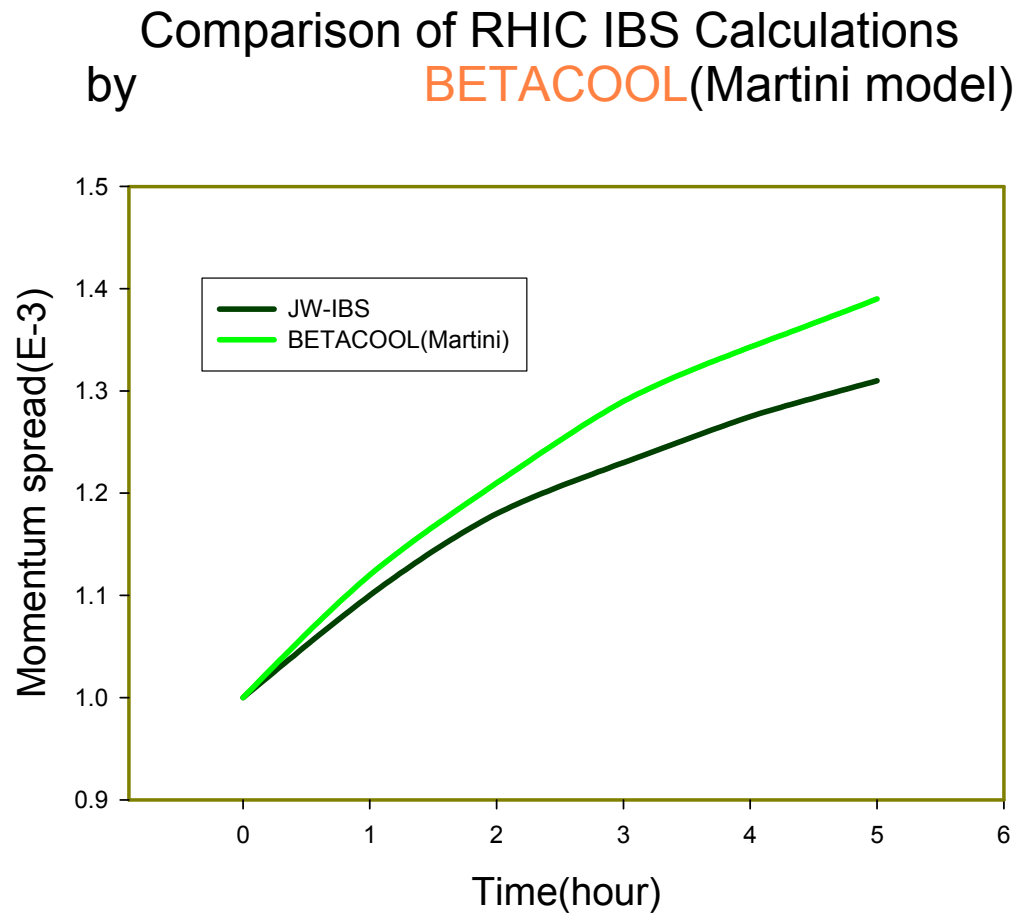

Figure 5 


\section{Summary}

The simulations show that

1) transverse emittance growth

Jie Wei's code gives the most pessimistic result, which is closer to the available experimental data so far. SIMCOOL gives the most optimistic results while BETACOOL(Martini model) is in between. The difference in transverse emittance after 5 hours is about $25 \% \sim 30 \%$.

2) Longitudinal aspect(bunch length and energy spread)

BETACOOL(Martini model) predicts a little bit worse results than Jie Wei's code does. However the difference is not significant.

Bunch length: the difference after 5 hours is about $4 \%$.

Momentum spread: the difference after 5 hours is about $6 \%$.

SIMCOOL does not include the synchrotron motion therefore no bunch length change is calculated while simulation.

\section{Acknowledgement}

The author would like to thank J. Wei and V. Parkhomchuk for providing their codes and offering enlightening discussions. Thanks also go to I. BenZvi for his thoughtful discussions and continuous encouragements. Anatoly Sidorin helped to fix the bugs in BETACOOL for IBS calculation during his visit in BNL.

\section{Reference}

[1] A. Piwinski, CERN Acc. School(1991) p.126

[2] J.D. Bjorken, S.K. Mtingwa, PA 13(1983) 115

[3] M. Martini, CERN PS/84-9(AA) 1984

[3] G. Parsen, NIM A256(1987) 231

[5] J. Wei, PAC 93, p.3651

[6] V. Parkhomchuk, I. Ben-Zvi, BNL C-A/AP/47(2001)

[7] I. Meshkov et al., BETACOOL code, JINR Interim report. 2002

[8] W. Fisher, et al., EPAC'02, p236-238, Paris, France, 2002 